\title{
Clinical and radiological midterm results from using the Fixion expandable intramedullary nail in transverse and short oblique fractures of femur and tibia
}

\author{
Carmine Zoccali • Alexander Di Francesco • \\ Alessandro Ranalletta $\cdot$ Stefano Flamini
}

Received: 15 September 2007 / Accepted: 27 May 2008/Published online: 2 August 2008

(C) Springer-Verlag 2008

\begin{abstract}
Background A locked nail is the principal method used to eliminate rotatory components in femoral and tibial fractures. Nevertheless, weight bearing is not directed onto the fracture site, slowing down the healing process; another possibility is to use a large-diameter nail and ream the canal to obtain as much adherence as possible and increase the grip, but this can cause a number of complications. The expandable nail is a new option that in theory should remove some problems with previous techniques.

Materials and methods This was a retrospective nonrandomized study encompassing 21 femoral fractures and 27 tibial fractures in 45 patients. They were classified according to the AO classification. Clinical and radiological checks were done at one, three, and six months and at one year from the surgery in order to check for signs of clinical and radiological healing. A good alignment was considered to be the presence of a deformity of less than $5^{\circ}$ in the sagittal and lateral planes and the absence of rotatory clinically evident problems. This protocol was adhered to up to six months after surgery by all of the patients, while
\end{abstract}

C. Zoccali - A. Di Francesco - A. Ranalletta - S. Flamini

"S. Salvatore" Regional Hospital of L'Aquila,

L'Aquila (AQ), Italy

C. Zoccali

Oncological Orthopaedics Department,

Muscular-Skeletal Tissue Bank IFO-IRE, Rome, Italy

C. Zoccali $(\bowtie)$

Università degli Studi dell'Aquila, Via Vetoio, 1, 67100 L'Aquila, Italy

e-mail: carminezoccali@libero.it

A. Ranalletta

University of L'Aquila, L'Aquila, Italy only $62.2 \%$ performed the last control. The mean follow-up was 15 months. A second group of 48 consecutive fractures ( 24 femural and 24 tibial) treated with locked nail was created to compare surgical times.

Results Appropriate alignment was observed in all cases; the healing process appeared slower: radiological healing occurred in most cases at six months. The following complications were reported: a case of intraoperative fracture widening with no effect on the treatment; a case of a lesion of the tip of the nail with pneumatic system rupture that necessitated nail substitution; two cases of retarded consolidation at six months, with both tibial fractures treated successfully by intralesion platelet gel; a case of incarcerated nail on 17 removals, resolved by shearing. We had no cases of clinically evident compartment syndrome or pulmonary embolism.

Conclusions The expandable Fixion nail presents significant advantages in the treatment of transverse and short oblique fractures of femur and tibia because it is easy to use, involves minimal X-ray exposure and can control rotations. Nevertheless, it high cost limits its use. We consider it as an alternative to locked nail.

Keywords Fixion nail - Expandable nail ·

Trasverse fracture osteosynthesis

\section{Introduction}

The system used in osteosynthesis directly influences fracture consolidation; depending on its shape and rigidity, osteosyntesis is capable of modifying bone callus formation. The intramedullary nail is currently the primary treatment for closed diaphyseal fractures and for grade I and II open lesions according to the Gustilo and Anderson 
classification $[1,2]$. This technique safeguards limb length and the soft tissue around the bone fragments. The immediate weight bearing allows gravity to act directly on the fracture site, encouraging bone callus formation.

Although the intramedullary nail is able to control dislocation and banding "ad latum," it is not able to control rotation [3]. Locking with proximal and distal screws impedes rotation so that it may be applied to metaphyseal fractures. However, this takes the weight off the fracture site, slowing down the healing process. Due to deformation of the nail during its insertion into the canal, the external guide may be not exact, forcing the surgeon to operate with excessive use of an X-ray image intensifier.

The use of a large-diameter nail is another possibility that should eliminate rotation and increase stability [4]. This involves reaming the canal. It has been reported that this action can cause numerous complications, such as intramedullary circulation injuries, a rise in local temperature, and increased risk of embolism and infection, although this is not in accordance with the literature [5,6]. One way to circumvent this problem could be to use the Fixion ${ }^{\circledR}$ expandable intramedullary nail (DISC-O-Teck, Herzliya, Israel). The diameter of this nail can be increased by $75 \%$ by introducing physiological solution inside it with a manual pump. In section, the expanded nail is characterized by four rods that penetrate the diaphyseal spongiosa. Its use removes the need for locking screws, and reaming becomes optional. Recent literature has emphasized the advantages of this technology for the surgeon (its ease of use) and for the patient (its good stabilization, and a healing rate of between 95 and 100\%) [7-16]. The aim of our study was to evaluate the use of this expandable intramedullary nail, focusing on indications, advantages, problems and midterm results from its application to femoral and tibial fractures.

\section{Materials and methods}

All cases of lower limb fractures observed in a regional general hospital from January 2003 to December 2005 that were treated with the Fixion expandable intramedullary stabilization system were evaluated. This cohort consisted of a consecutive series of operations performed by the senior author (S.F.). Inclusion criteria were femoral consecutive fractures classified according to the AO classification as A2, A3, B1, B2 and tibial consecutive fractures of types A2, A3, B2 and B3 [17]. Other treatments were performed for long oblique and comminuted fractures, and for Gustilo and Anderson type II and III fractures.

The cases were classified as follows: 45 patients (aged from 17 to 58 years, mean 35), 48 inferior limb fractures, of which 21 were femoral fractures and 27 tibial fractures; three patients had been treated for both femoral fracture and tibial fracture. Our fracture series was distributed as follows: 11 fractures of type $\mathrm{A} 2$, three fractures of type $\mathrm{A} 3$, three fractures of type B1 and two of type B2. Our tibial fracture series was distributed as follows: seven fractures of type $\mathrm{A} 2,16$ fractures of type $\mathrm{A} 3$, one fracture of type $\mathrm{B} 2$ and three of type B3.

We observed only one case of Gustilo and Anderson type I open fracture. All operations had been performed within $72 \mathrm{~h}$ of the trauma. Short-term antibiotic prophylaxis with cephalosporins was carried out; in cases with a high risk of infection, teicoplanin $(400 \mathrm{mg})$ was added. Prevention of deep-vein thrombosis was achieved with low-molecular weight heparin, starting at the moment of trauma and continuing for three weeks or until the patient had regained adequate ambulation.

The nail was introduced via trochanteric access for femoral fractures and by transpatellar tendon for tibial fracture; in four cases the medullary canal was reamed. Nail length was determined using a metallic rule and an Xray image intensifier.

Post-operative management: in the first post-operative day, active and passive physiotherapy of the neighboring joints was initiated without the use of a tutor to maintain muscular tone. Weight bearing was permitted on the second post-operative day using two crutches or a Zimmer frame; partially at first, and then progressively starting from ten days after surgery. X-ray controls were performed at one, three and six months after surgery for all patients, and at one year for 28 patients because of most of them lived too far away. The mean follow-up was 15 months.

Nails were removed at one year if possible; later removal is very difficult because of bone integration, so that attempts were only made after one year in young people. A second control group was created by selecting 48 consecutive patients previously treated with conventional locked nails for similar types of femoral and tibial fractures. This second group was similar to the first one in terms of demographic distribution (age 23-62 years, mean 37) and fracture type. It consisted of 24 femoral fractures (16 A2, 1 A3, 4 B1, 3 B2) and 24 tibial fractures (9 A2, 14 A3, 1 B3).

Surgical time was calculated from incision until skin suture and confront was done using a parametric test for paired data (Student's $t$ test).

The study was approved by the local ethics committee and conducted according to the Declaration of Helsinki; the patients gave their informed consent prior to inclusion.

\section{Results}

Average surgical time in the Fixion nail group was $55 \mathrm{~min}$ (range, 35-90; SD, 12.5) versus $74 \mathrm{~min}$ in the control group (range, 50-110; SD, 14.09). This difference is statistically 
significant $(P<0.0001)$. Postoperative checks evidenced good alignment in all cases with a good rotation and banding control: no angulation of $>5^{\circ}$ or clinically evident rotatory deformity was observed. Shortening was evaluated clinically by referring to the other limb: a shortening of $>2 \mathrm{~cm}$ was observed in two cases of femoral fracture (1B1, 1B2) and in one case of tibial fracture (1B3); nevertheless, information about the pre-injury state was not available. The healing process appeared to be slower: radiological healing, that is the presence of bone bridges in at least three of the four corticals arose in three-quarters of the cases at the sixmonth control and in only one-quarter of them previously.

We report the following complications here: a case of intraoperative fracture widening with no effect on the treatment; a lesion of the tip of the nail with pneumatic system rupture that necessitated nail substitution; two cases of retarded consolidation at six months, where both tibial fractures were treated successfully with intralesion platelet gel; a case of nail incarceration on 17 removals, resolved by shearing. No cases of clinically evident compartment syndrome or pulmonary embolism were observed.

\section{Discussion}

The intramedullary nailing story started in the 1950s with the Kuntscher nail. Using a large-dimension nail after canal
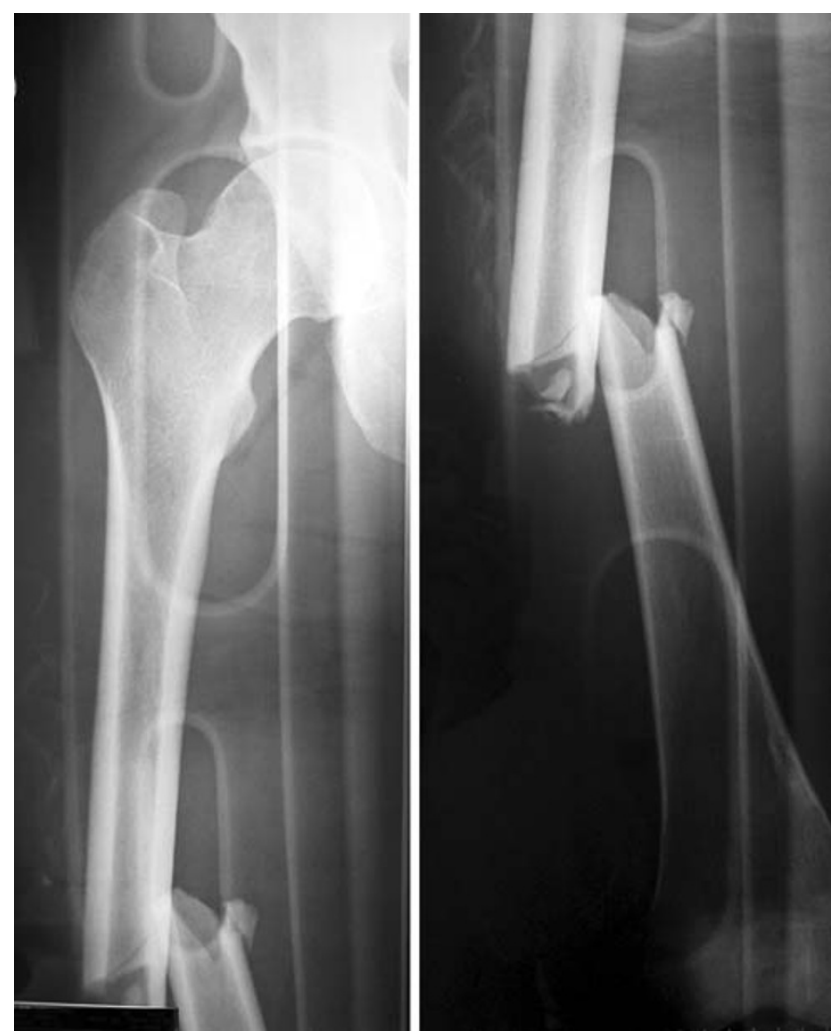

Fig. 1 Male, 32 years old: preoperative X-rays showing a type A3 fracture

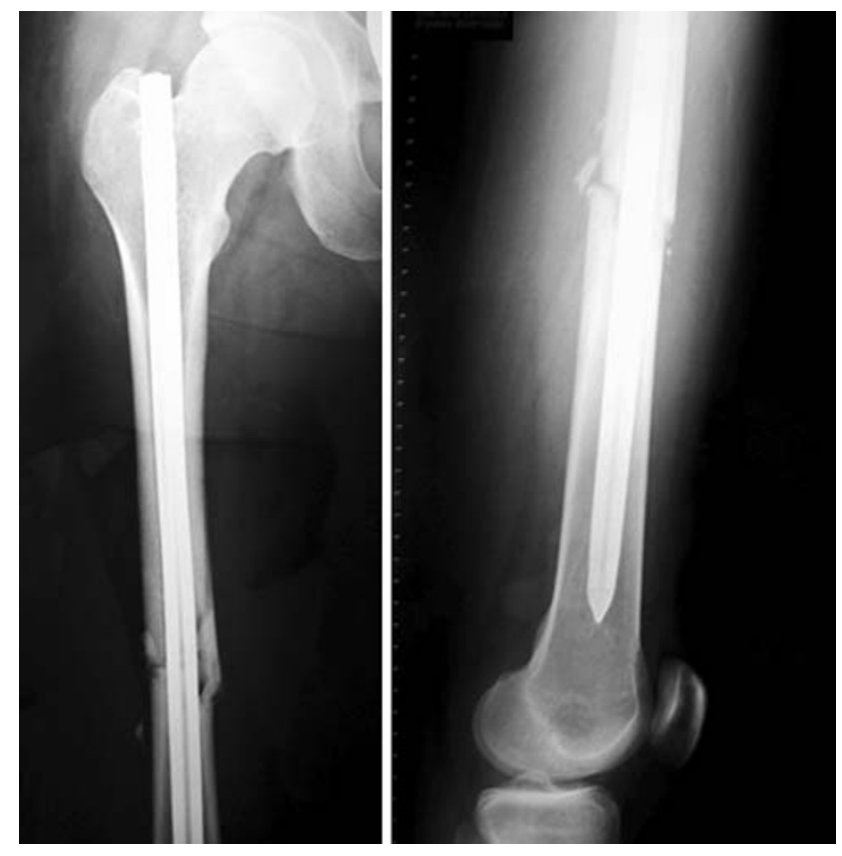

Fig. 2 One-month postoperative X-rays

reaming was mandatory in order to guarantee rotatory stability [18]. However, this option was (and still is) associated with complications such as intramedullary

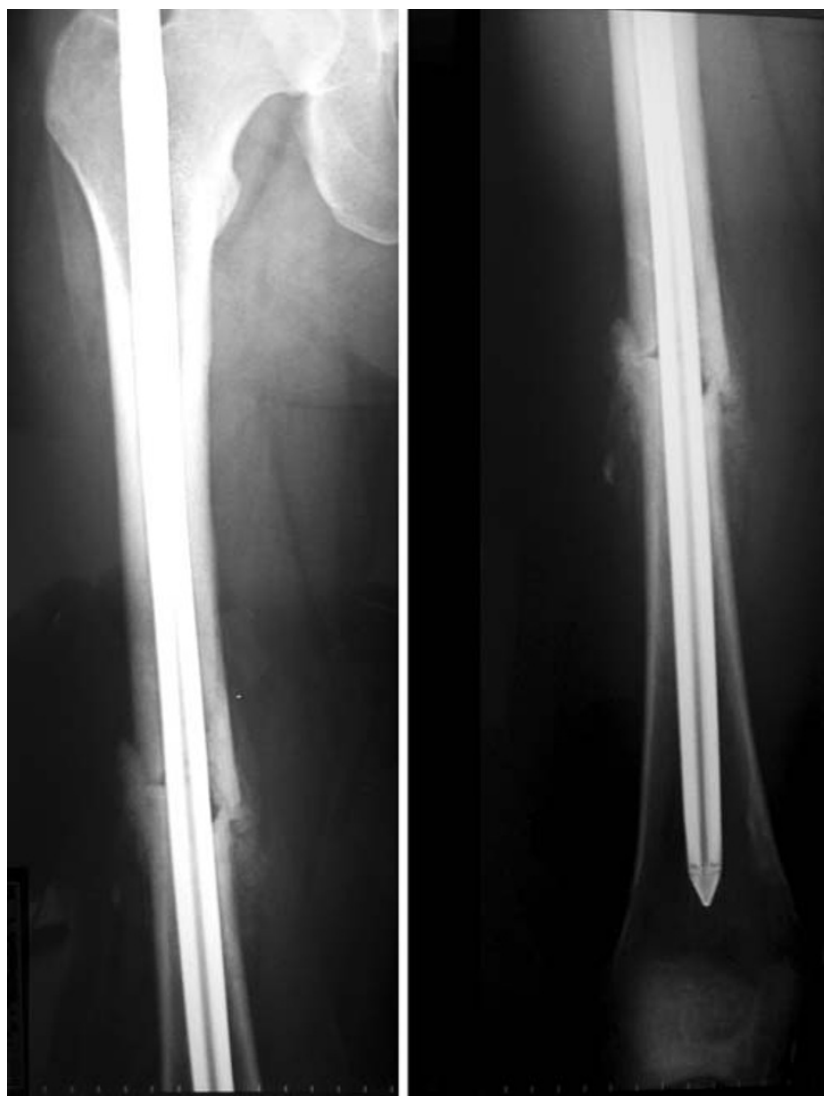

Fig. 3 Three-month postoperative X-rays 


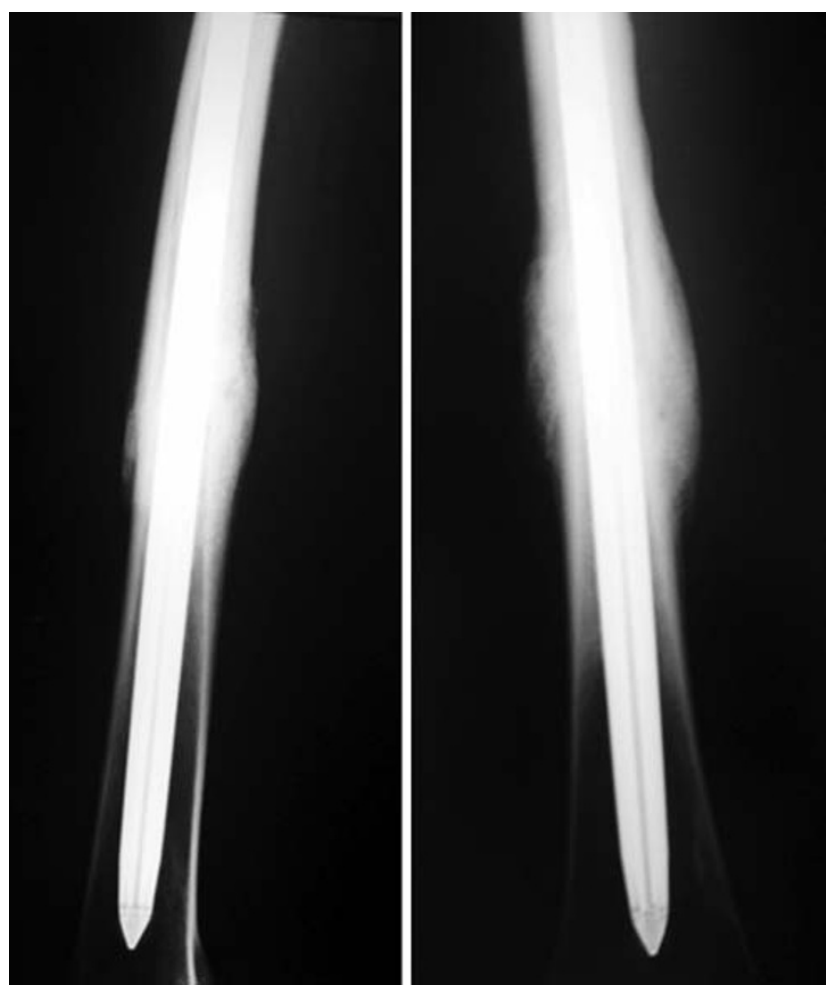

Fig. 4 Six-month postoperative X-rays showing the radiological healing process

material embolism (particularly dangerous in polytraumatism with respiratory distress) and an increased risk of infection, particularly in open fractures. Furthermore, associated intramedullary circulation damage causes a decrease in endosteal callus [19-21].

The expandable Fixion nail may be inserted without reaming, ensuring rotatory stability, just as for bigger nails. However, it is necessary to evaluate if the high contact pressure inside the intramedullary canal slows down the healing process. This would explain the retarded radiographic healing observed in our series. Difficulty with performing distal locking is a problem in intramedullary locked stabilization. This is particularly evident in the femur more than in the tibia: because of the "procurvatum" present in the femoral shape, the nail inserted into the canal is subject to slight deformation, so that the external guide becomes less efficient, forcing the surgeon to operate using an X-ray image intensifier; this is potentially dangerous for him, the patient and for other people involved [22]. The use of the expandable intramedullary nail can circumvent this problem. An average decrease of $20 \mathrm{~min}$ in intraoperative time was observed because of it is easier to introduce and there is no locking time. It is important to avoid damaging the expansion system, which is particularly frail at the tip, in order to avoid the need to change the nail. Because of its characteristics, it is appropriate for treating femoral fractures classified as A2, A3, B1 and B2 and localized $7 \mathrm{~cm}$ distally from the lesser trochanter and $15 \mathrm{~cm}$ proximally to the knee joint rim. We consider type A1 and B3 fractures to be contraindications: type A1 because of the oblique borders and and type B3 because it is a multifragmentated fracture and the risk of limb shortening is too high. A
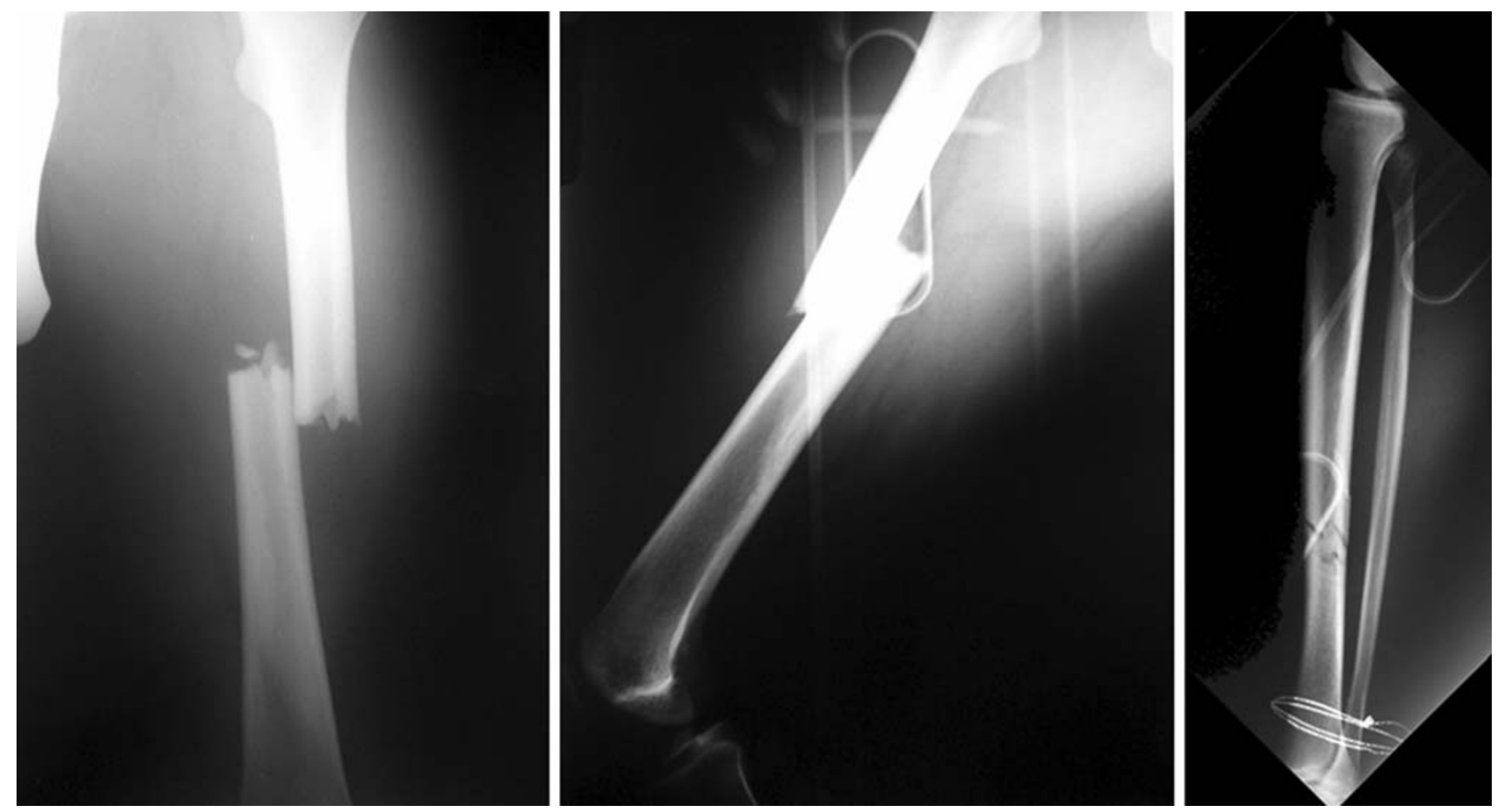

Fig. 5 Male, 41 years old: preoperative X-rays showing a type A3 femoral fracture and a type A3 tibial fracture 

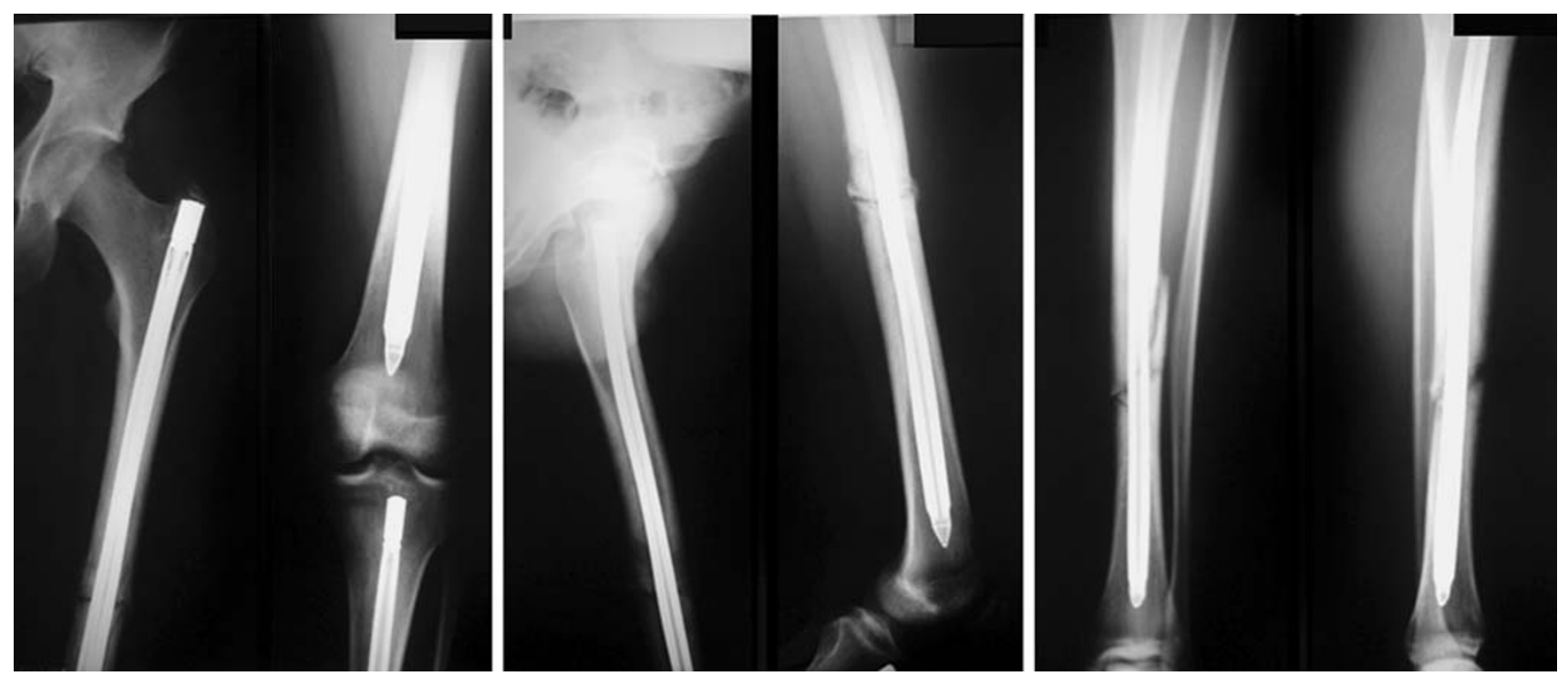

Fig. 6 One-month postoperative X-rays
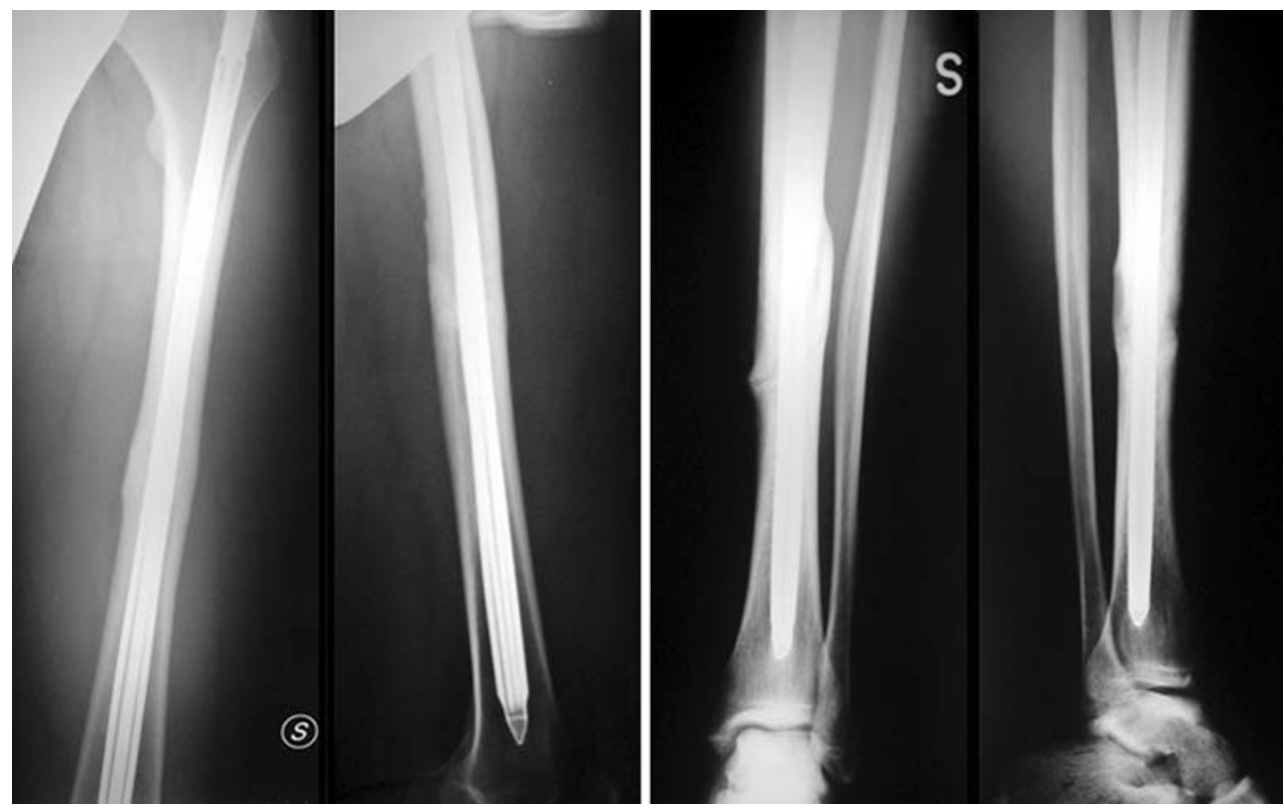

Fig. 7 Three-month postoperative X-rays

representative case of femoral fracture is shown (Figs. 1, 2, $3)$. In the tibia we used the expandable nail for types A2, $\mathrm{A} 3, \mathrm{~B} 2$ and $\mathrm{B} 3$, localized $7 \mathrm{~cm}$ distally from the tibial tuberosity and $7 \mathrm{~cm}$ proximally to the distal canal end; we did not treat A1 and B1 fractures because they were oblique. A second case of both femoral and tibia fracture is also shown (Figs. 4, 5, 6, 7). In our series, the results were considered satisfactory; evident problems concerning limb shortening that forced Wade et al. [23] to stop their prospective study were not observed.
Further studies are needed to evaluate the clinical and radiological healing times, which were increased in our series, and the importance of limb shortening for type AO fractures, considering that in the abovementioned study the authors used the expansion system for type A1 femoral fractures as well as for type A1 and B1 tibial fractures. All possible advantages should be taken into account when considering the elevated cost of the Fixion nail. Its use may be considered as a second choice for transverse and short oblique fractures in femur and tibia. 
Conflict of interest statement The authors declare that they have no conflict of interest related to the publication of this manuscript.

\section{References}

1. Broos PL, Sermon A (2004) From unstable internal fixation to biological osteosynthesis. A historical overview of operative fracture treatment. Acta Chir Belg 104(4):396-400

2. Finkemeier CG, Schmidt AH, Kyle RF, Templeman DC, Varecka TF (2000) A prospective, randomized study of intramedullary nails inserted with and without reaming for the treatment of open and closed fractures of the tibial shaft. J Orthop Trauma 14(3):187-93

3. Kuntscher G (1965) Intramedullary surgical technique and its place in orthopaedic surgery. My present concept. J Bone Joint Surg Am 47-A:809-818

4. Kyle RF (1985) Biomechanics of intramedullary fracture fixation. Orthopedics 8(11):1356-1359

5. Ma S, Wang K, Zhang M (2006) Comparative study on the effect of reamed and nonreamed intramedullary nails on treating open tibial fractures. Zhongguo Xiu Fu Chong Jian Wai Ke Za Zhi 20(1):23-26

6. Wenda K, Runkel M (1996) Systemic complications in intramedullary nailing. Orthopade 25(3):292-299

7. Bekmezci T, Tonbul M, Kocabaş R, Yalaman O (2004) Early treatment results with expandable intramedullary nails in lower extremity shaft fractures. Ulus Travma Acil Cerrahi Derg 10(2):133-7

8. Capelli RM, Galmarini V, Molinari GP, De Amicis A (2003) The Fixion expansion nail in the surgical treatment of diaphyseal fractures of the humerus and tibia. Our experience. Chir Organi Mov 88:57-64

9. Franck WM, Olivieri M, Jannasch O et al. (2002) An expandable nailing system or the management of pathologic humerus fractures. Arch Orthop Trauma Surg 122:400-405

10. Franck WM, Olivieri M, Jannasch O, Hennig FF (2003) Expandable nail system for osteoporotic humeral shaft fractures: preliminary results. J Trauma 54:1152-1158

11. Jovanovic A, Pirpiris M, Semirli H, Doig SG (2004) Fixion nails for humeral fractures. Injury 35:1140-1142
12. Lepore L, Lepore S, Maffulli N (2003) Intramedullary nailing of the femur with an inflatable self-locking nail: comparison with locked nailing. J Orthop Sci 8:796-801

13. Lepore S, Capuano N (2000) Preliminary clinical and radiographic results with the Fixion intramedullary nail: an inflatable self-locking system for long bone fractures. J Orthopaed Traumatol 3:135-140

14. Panidis G, Sayegh F, Beletsiotis A et al. (2003) The use of an innovative inflatable self-locking intramedullary nail in treating and stabilizing long bone fractures. Technique-preliminary results. Osteo Trauma Care 11:S108-S112

15. Pascarella R, Nasta G, Nicolini M, Bertoldi E, Maresca A, Boriani S (2002) The Fixion nail in the lower limb. Preliminary results. Chir Organi Mov 87:169-174

16. Shasha N, Blumberg N, Tauber M et al. (eds) (2002) An expandable intramedullary nail for fixation of osteoporotic bone. Internal fixation in osteoporotic bone. Thieme Verlagsgruppe, New York

17. Müller ME, Nazarian S, Koch P et al. (1990) The comprehensive classification of fractures of long bones. Springer, Berlin

18. Fairbank AC, Thomas D, Cunningham B, Curtis M, Jinnah RH (1995) Stability of reamed and unreamed intramedullary tibial nails: a biomechanical study. Injury 26(7):483-485

19. Blachut PA, O’Brien PJ, Meek RN, Broekhuyse HM (1997) Interlocking intramedullary nailing with and without reaming for the treatment of closed fractures of the tibial shaft. A prospective, randomized study. J Bone Joint Surg Am 79(5):640-646

20. Koval KJ, Clapper MF, Brumback RJ, Ellison PS Jr, Poka A, Bathon GH, Burgess AR (1991) Complications of reamed intramedullary nailing of the tibia. J Orthop Trauma 5(2):184-189

21. Orler R, Locher S, Lottenbach M, Heini P, Ganz R (2002) Retrospective analysis of healing problems after reamed and unreamed nailing of femoral shaft fractures. Unfallchirurg 105(5):431-436

22. Whatling GM, Nokes LD (2006) Literature review of current techniques for the insertion of distal screws into intramedullary locking nails. Injury 37(2):109-119

23. Smith WR, Ziran B, Agudelo JF, Morgan SJ, Lahti Z, Vanderheiden T, Williams A (2006) Expandable intramedullary nailing for tibial and femoral fractures: a preliminary analysis of perioperative complications. J Orthop Trauma 20(5):310-314 